- Presents the salient clinical features of primary herpetic gingivostomatitis and outlines supportive therapy.

- Describes persistent labial adhesions following primary herpetic gingivostomatitis infection.

- Alerts the profession to a complication which is potentially preventable by the use of barrier creams in the acute phase of primary herpetic gingivostomatitis.

\title{
A complication of primary herpetic gingivostomatitis
}

\author{
E. Thomas ${ }^{1}$
}

A complication of primary herpetic gingivostomatitis and its treatment is described in a 7-year-old child. A reminder to recommend lip barrier creams is advised to prevent this complication pain and bleeding when the lips are separated following adhesion.

\section{INTRODUCTION}

Primary herpetic gingivostomatitis (PHGS) is the most common clinical manifestation of primary herpes simplex virus infection occurring in $25-30 \%$ of infected children. ${ }^{1}$ Symptoms including discomfort on swallowing and excessive salivation, submandibular lymphadenopathy, oral and peri oral ulceration frequently occur.

Patients often seek advice from their GDP who will advise supportive management including maintaining fluid intake to prevent deyhdration; analgesics and antipyretics to ease fever and discomfort; and the application of topical antiviral preparations to limit the duration of symptoms. ${ }^{2}$ It is not now common practice to advise the use of lip barrier cream to prevent adherence of the lips, pain and the bleeding often caused from their separation. This paper presents a rare complication from PHGS

Maxillofacial Surgery/Paediatric Dentistry, Arrowe Park Hospital, Upton, Wirral, Merseyside, CH49 5PE

Correspondence to: Dr Elin Thomas

Email: elinthomasina@yahoo.co.uk

\section{Refereed Paper}

Accepted 17 October 2006

DOI: $10.1038 /$ bdj.2007.585

${ }^{\odot}$ British Dental Journal 2007; 203: 33-34 and reintroduces the need for barrier creams as standard advice to patients with the condition.

\section{CASE REPORT}

A 7-year-old girl was referred to a Consultant Paediatrician at Arrowe Park Hospital with a two week history of fever, lymphadenopathy, oral and peri oral ulceration. A diagnosis of PHGS was made on clinical criteria. She returned two weeks following recovery with labial adhesions which were limiting mouth opening and creating social embarrassment, and was referred to the Oral and Maxillofacial Surgery Department. She had bilateral labial adhesions extending from the labial commisures across the lips (Fig. 1). No active treatment was instigated as it was hoped that the adhesions would be broken during function. At two week review the lesions persisted so they were released under general anaesthesia. The patient made an uneventful recovery with no lip deformity or visible scarring.

\section{DISCUSSION}

PHGS is caused by the DNA herpes simplex virus (HSV) with HSV type 1 mainly associated with infections of the skin and mucous membranes, and type
2 implicated in genital infections, ${ }^{3}$ but this distinction is increasingly becoming less clear. ${ }^{4}$ Type 1 infection is common worldwide and increases in prevalence to $70-80 \%$ in adulthood. ${ }^{5}$

Primary HSV infection in childhood is usually subclinical, ${ }^{2}$ or it may cause a mild pharyngitis ${ }^{1}$ with PHGS typically affecting $25-30 \%$ of infected children. ${ }^{3}$

Transmission is from infected oral secretions with an incubation period of 1-26 days followed by prodromal symptoms including malaise, anorexia, nausea, irritability, headache, fever, submandibular lymphadenopathy with later the appearance of numerous characteristic oral vesicles. The vesicles rupture readily leaving ragged painful ulcers covered by a yellow-grey pseudomembrane. These clinical findings are diagnostically accurate in $83.8 \%$ of cases. ${ }^{6}$

PHGS infection may cause dehydration necessitating hospital admission while recurrent HSV infections range from inoccuous labial lesions to life threatening meningoencephalitis. ${ }^{7}$

PHGS is a self limiting infection, however antiviral therapy may reduce the duration and severity of symptoms. ${ }^{8}$ Oral acyclovir suspension is the current treatment but the optimal timing and dose of this therapy are 


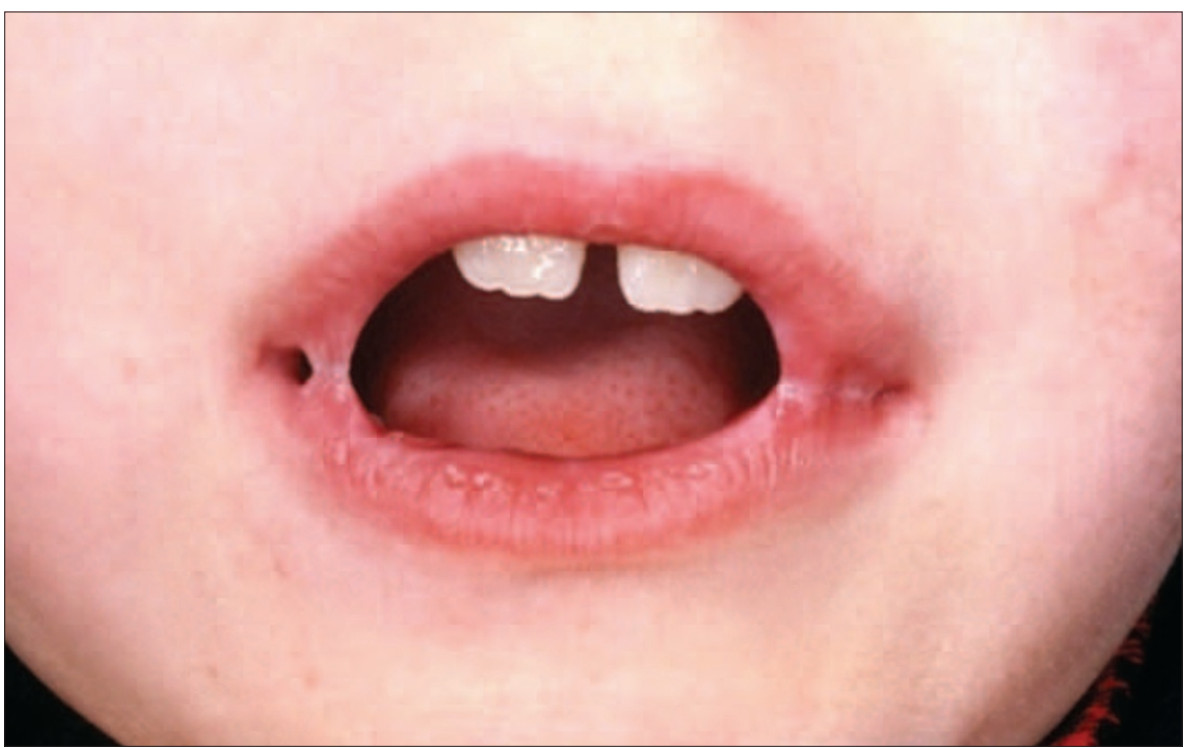

Fig. 1 Labial epithelisation as a result of adherent PHGS ulcer healing.

uncertain. ${ }^{8}$ Supportive therapy is aimed at reducing complications and the application of lip barrier cream would have prevented the labial adhesions in the presented case. This simple yet effec-
With thanks to Mr G. D. Wood, Consultant Oral and Maxillofacial Surgeon, for allowing me to present his case and for encouraging me to write this report.

1. Cesario T C, Poland J D, Wulff H et al. Six years experience with herpes simplex virus in a childern's home. Am J Epidemiol 1969; 90: 416-422.

2. Arduino P G, Porter S R. Oral and perioral herpes simplex virus type 1 (HSV-1) infection: review of its management. Oral Dis $2006 ; 12: 254-270$.

3. Soames J V, Southam J C. Oral pathology. pp 183186. Oxford University Press, 1998.

4. Nieuwenhuis R F, van Doornum G J, Mulder P G et al. Importance of herpes simplex virus type-1 in primary genital herpes. Acta Derm Venereol 2006. 86: 129-134.

5. Stock C, Guillan-Grima F, Hermosa de Mendoza J et al. Risk factor of herpes simplex type I (HSV-1) infection and lifestyle factors associated with HSV-1 manifestations. Eur J Epidemiol 2001; 17: 885-890.

6. Amir J, Straussberg R, Harel L et al. Evaluation of a rapid enzyme immunoassay for the detection of herpes simplex antigen in children with herpetic gingivostomatitis. Pediatr Infect Dis J 1996: 15: 627-629.

7. Kolokotronis A, Doumas S. Herpes simplex virus infection, with particular reference to the progression and complications of primary herpetic gingivostomatitis. Clin Microbial Infect 2006; 12: 202-211.

8. Arduino P G, Porter S R. Oral and perioral herpes simplex virus type 1 (HSV-1) infection: review of its management. Oral Dis 2006; 12: 254-257. 